\title{
INEQUALITIES FOR ZEROS OF SOLUTIONS TO SECOND ORDER ODE WITH ONE SINGULAR POINT
}

\section{MiCHAEL GIL'}

Abstract. We consider the equation $y^{\prime \prime}+P(z) y^{\prime}+Q(z) y=0 \quad(z \in \mathbb{C})$, where

$$
P(z)=\sum_{k=0}^{n_{P}} p_{k} z^{k-1} \text { and } Q(z)=\sum_{k=0}^{n_{Q}} q_{k} z^{k-2}
$$

with real coefficients $p_{k}, q_{j}\left(k=0, \ldots, n_{P} ; j=0, \ldots, n_{Q} ; n_{P}, n_{Q}<\infty\right)$.

Let $z_{k}(y), k=1,2, \ldots$ be the nontrivial zeros of a solution $y(z)$ to that equation. Estimates for the sums $\sum_{k=1}^{j} \frac{1}{\left|z_{k}(y)\right|}(j=1,2, \ldots)$ are derived. Applications of the obtained estimates to the counting function of the zeros of solutions are also discussed.

Mathematics subject classification (2010): 34C10, 34A30. free domain.

Keywords and phrases: ODE with a singular point, complex zeros of solutions, counting function, zero

\section{REFERENCES}

[1] N. Anghel, Stieltjes-Calogero-Gil relations associated to entire functions of finite order, J. of Mathem. Phys., 51, no. 5 (2010) 251-262.

[2] B. BELAIDI, Oscillation of fast growing solutions of linear differential equations in the unit disc, Acta Univ. Sapientiae Mathematica, 2, no. 1 (2010), 25-38.

[3] T. B. CAO AND H. X. YI, On the complex oscillation theory of linear differential equations with analytic coefficients in the unit disc, Acta Math. Sci., 28A (6) (2008), 1046-1057.

[4] T. B. CAO, LIU KAI AND XU HONG-YAN, Bounds for the sums of zeros of solutions of $u(m)=P(z) u$ where $P$ is a polynomial. Electron. J. Qual. Theory Differ. Equ. no. 60, (2011), 10 pp.

[5] Yu L. Daleckil AND M.G. Krein, Stability of Solutions of Differential Equations in Banach Space, Amer. Math. Soc., Providence, R. I. 1971.

[6] M.I. GIL', Localization and Perturbation of Zeros of Entire Functions, CRC Press, Taylor and Francis Group, New York, 2010.

[7] M.I. GIL', Bounds for zeros of solutions of second order differential equations with polynomial coefficients, Results Math., 59 (2011), 115-124.

[8] M.I. GIL', Perturbation of zeros of solutions to second order differential equations with polynomial coefficients, Acta Matematica Scientia, 32 (3), (2012), 1083-1092.

[9] M.I. GIL', Sums of zeros of solutions to second order ODE with non-polynomial coefficients, Electron. J. Diff. Equ., Vol. 2012 (2012), no. 107, 1-8.

[10] M.I. GIL', Sums of zeros of solutions to non-homogeneous ODE with polynomial coefficients, J. Math. Anal. Appl. 421, no. 2, (2015), 1917-1924.

[11] E. HiLle, Lectures on Ordinary Differntial Equations, Addison Wesley Publiching Company, Ontario, 1969.

[12] E.L. InCE, Ordinary Differential Equations, Dover Publ., New York, 1978.

[13] I. Laine, Nevanlinna Theory and Complex Differential Equations, Walter de Gruyter Berlin, 1993

[14] Z. Latreuch, B. Belaidi AND A. El Farissi, Complex oscillation of differential polynomials in the unit disc, Periodica Mathematica Hungarica, 66 (1), (2013), 45-60. 
[15] G.M. Muminov, On the zeros of solutions of the differential equation $\omega^{(2 m)}+p(z) \omega=0$. Demonstr. Math., 35, no. 1 (2002), 41-48.

[16] F. PENG AND Z. X. CHEN, On the growth of solutions of some second-order differential equations, J. Ineq. Appl., 2011 (2011), 1-9.

[17] J. TU AND Z. X. CHEN, Zeros of solutions of certain second order linear differential equation, J. Math. Anal. Appl. 332, no. 1 (2007), 279-291.

[18] J. F. XU AND H. X. YI, Solutions of higher order linear differential equations in an angle, Appl. Math. Letters, 22, no. 4 (2009), 484-489. 